\title{
Temperature Profiles in Hamiltonian Heat Conduction
}

\author{
Jean-Pierre Eckmann ${ }^{1,2}$ and Lai-Sang Young ${ }^{3}$ \\ ${ }^{1}$ Département de Physique Théorique, Université de Genève \\ ${ }^{2}$ Section de Mathématiques, Université de Genève \\ ${ }^{3}$ Courant Institute for Mathematical Sciences, New York University
}

\begin{abstract}
We study heat transport in the context of Hamiltonian and related stochastic models with nearest-neighbor coupling, and derive a universal law for the temperature profiles of a large class of such models. This law contains a parameter $\alpha$, and is linear only when $\alpha=1$. The value of $\alpha$ depends on energy-exchange mechanisms, including the range of motion of tracer particles and their times of flight.

PACS numbers: 05.70.Ln, 05.45.-a
\end{abstract}

In nonequilibrium physics, the Fourier law is an example of a simple phenomenological principle whose molecular origin is very hard to explain. Idealizing homogeneous thin rods and wires with uniform cross-section as 1-D objects, this law says that heat flux is proportional to temperature gradient times heat conductivity. Ever since Fourier's pioneering work [1], physicists have tried to derive this law from first principles. The current state of the art is summarized in the excellent reviews [2, 3, 4], which all point to the need for a deeper theoretical understanding beyond the many existing models and simulations. In this Letter, we report on new results for certain types of Hamiltonian systems and their stochastic realizations.

To study the Fourier law in a Hamiltonian context, the most common setting is that of a chain of identical units comprised of disks, plates, penduli, and the like, coupled with short range forces between them. At its two ends, the chain is coupled to mechanisms simulating heat baths maintained at two different temperatures. Because the components of the chain are identical, one may expect heat conductivity to be constant along the chain, so that by Fourier's law, the temperature profile is linear. This seems to be the predominant thinking behind much of the recent work on Fourier's Law, although nonlinear profiles are known to occur in other contexts [5].

In this Letter we point out some simple and natural mechanisms that lead to various profiles - both linear and nonlinear - in concrete Hamiltonian models, and derive a universal law for the profiles of these and other systems.

\section{Summary of results:}

(A) We consider Hamiltonian models consisting of a chain of energy storing devices (ESD) that are fixed in place and coupled to each other. For such a setup, we show that the temperature profile can be linear or nonlinear depending on the nature of the coupling. More specifically, we assume that energy exchange in the system is mediated by tracers, which move from ESD to ESD redistributing energy according to the rules introduced in [6, 7]. We find that the profile is linear if energy transfer is carried out by a single tracer that moves freely along the chain, whereas heat conductivity is temperature-dependent (and hence the profile is nonlinear) if the tracers are confined to specific regions.

(B) Our second result is a universal law that holds for very general coupled chains of Hamiltonian or stochastic systems with nearest-neighbor interaction (including those considered in (A)). We show that as the number of constituent cells goes to infinity, the stationary temperature profile is given by

$$
T(x)=\left(T_{\mathrm{L}}^{\alpha}+\left(T_{\mathrm{R}}^{\alpha}-T_{\mathrm{L}}^{\alpha}\right) x\right)^{1 / \alpha}
$$

where $T_{\mathrm{L}}$ and $T_{\mathrm{R}}$ are the temperatures imposed at the left and right ends, $x$ is the coordinate along the system (normalized to $x \in[0,1])$, and $\alpha$ is a constant. The form of this law does not depend on details of the system (precise conditions are given later). The value of $\alpha$ in (1), however, depends on the nature of the coupling.

In the case of locked-in tracers, i.e., tracers confined to the regions between neighboring ESD, the value of $\alpha$ depends on their time of flight. In particular, $\alpha=\frac{3}{2}$ if the energy is purely kinetic. In the case of a single tracer moving freely along the chain, $\alpha=1$ independent of its time of flight. We show the results above for stochastic realizations of the models in (A), and explain why one should expect these results to carry over to their Hamiltonian counterparts.

Our primary concern in this Letter is the temperature profile (TP). The existence of local thermal equilibrium (LTE), which is important for the definition of temperature, will be proved in [8] in the infinite volume limit for one of the models treated here. (For results on LTE for other models, see [9, 10, 11].)

Before embarking on specifics, we note again that in our models, we distinguish between communicating agents (CA), which in our case are tracer particles, and ESD, which in our case are turning disks with fixed centers (we emphasize that the ESD are not infinite reservoirs). In a real-world conductor, the difference between CA and ESD is often blurred, but for a good theoretical understanding it is useful to keep them separate. These concepts are distilled from the following beautiful model:

The MLL Model [6, 7]: We describe this model in some detail, as it contains the basic ingredients of the models in (A). The MLL Model is purely Hamiltonian, and very careful simulations show that the Fourier law holds. The system consists of an arrangement of $N$ disks of radius 1 placed as in Fig 1 and a little point particle of mass 1 (the tracer) which wanders around the playground $\Omega$ (the physical space occupied by the system minus the disks), bouncing off the disks [17]. While Fig 1 suggests a Lorentz gas [12], there is a crucial dif- 


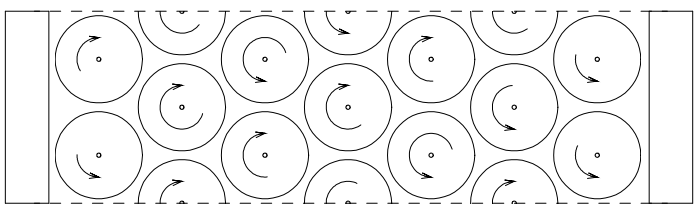

FIG. 1: A typical arrangement of disks in the MLL Model. The simulations in [7] were done with 2 rows and periodic boundary conditions in the vertical direction, and zig-zag reflecting walls of temperature $T_{\mathrm{L}}$, resp. $T_{\mathrm{R}}$, at the two ends. The tracer particle is not shown.

ference here: Each disk is "nailed down" in its center, around which it turns freely. The state of the system is described by $x=\left(\omega_{1}, \ldots, \omega_{N}, q, v\right)$ where $\omega_{i}$ is the angular velocity of disk $i, q \in \Omega$ is the position of the tracer, and $v$ is its velocity. When the tracer collides with a disk, the rule of interaction is that of "sticky reflection": Suppose the angular velocity of the disk being hit is $\omega$, and $v_{\mathrm{n}}$ and $v_{\mathrm{t}}$ are the normal, resp. tangential, component of $v$ relative to the impact point. Then the values of $v$ and $\omega$ after the collision are given by the energy and angular momentum conserving law

$$
\begin{aligned}
v_{\mathrm{n}}^{\prime}=-v_{\mathrm{n}}, \quad v_{\mathrm{t}}^{\prime} & =v_{\mathrm{t}}-\frac{2 \varepsilon}{1+\varepsilon}\left(v_{\mathrm{t}}-\omega\right), \\
\omega^{\prime} & =\omega+\frac{2}{1+\varepsilon}\left(v_{\mathrm{t}}-\omega\right) .
\end{aligned}
$$

Here, $\varepsilon \in \mathbf{R}^{+}$is proportional to the moment of inertia of the disk divided by the mass of the tracer and the square of the radius of the disk. [7] treats mostly the case $\varepsilon=1$, where $v_{\mathrm{t}}^{\prime}=$ $\omega$ and $\omega^{\prime}=v_{\mathrm{t}}$, i.e., the two quantities are simply exchanged. Of particular interest to us is the case $\varepsilon \ll 1$, which from the tracer's point of view resembles the classical Lorentz gas.

Remark. The MLL Model, as well as the Hamiltonian models we will describe later, have the following important property: Since there is only a hard-core potential, the time evolution of the system is rescaled by $\sqrt{\lambda}$ when the energy of the particle and the disks are rescaled by $\lambda$. In this respect, the model in [7] is very different from models such as the dinga-ling and ding-dong models [13, 14, 15]. Most importantly, the energies of the tracer and the disks alone determine the time-of-flight of the tracer: It does not depend on the history (as it would in many models considered so far [13, 14, 15]).

We still need to say what happens when the tracer hits one of the ends. In [7], many variants are considered, but for our purpose, the following process is assumed: When the tracer hits one of the ends, it exits the system, and a new tracer is injected into $\Omega$ to take its place. The new tracer enters $\Omega$ at the point of exit of the old one. Its direction is arbitrary, and its speed is given by the Maxwell distribution for the temperature of the end in question.

Introducing the models studied in this Letter: We now introduce two classes of models that have the same basic setup of tracers and turning disks (and the same rules of interaction) as in the MLL Model. However, the configurations of disks and tracers in these two models are chosen to give rise to two conceptually very different modes of transport.

Model I (Wandering tracer): A single tracer wanders along a chain of boxes separated by walls with a tiny hole that allows the tracer to pass between adjacent boxes. Deep inside each box is a turning disk surrounded by many fixed disks. The turning disk serves as ESD, while the fixed disks are bona fide Lorentz scatterers, which serve to randomize the angles of incidence in collisions between the tracer and the turning disk, leading to the exchange of a random portion (i.e., the tangential component) of the energy of the tracer. The smallness of the holes in the separating walls keeps the tracer in each box for a long period. This together with the chaotic action of the Lorentz scatterers ensures that the tracer is equally likely to exit the box from either side.

Stochastic realization: We model this system on a 1-D lattice with $N$ sites. There is a random variable $\xi_{i}$ representing the energy at site $i, i=1, \ldots, N$, with values in $[0, \infty)$. The heat baths at the ends are modeled by stochastic variables $\xi_{\mathrm{L}}$ and $\xi_{\mathrm{R}}$ which take values in $[0, \infty)$ with a distribution $T_{\mathrm{L}} \exp \left(-\xi_{\mathrm{L}} / T_{\mathrm{L}}\right)$, resp. $T_{\mathrm{R}} \exp \left(-\xi_{\mathrm{R}} / T_{\mathrm{R}}\right)$ (the Boltzmann constant $k_{\mathrm{B}}$ being set to 1$)$. We identify $\xi_{\mathrm{L}}$ with the variable $\xi_{0}$, and $\xi_{\mathrm{R}}$ with $\xi_{N+1}$. There are two more random variables, $\eta$, to be thought of as the energy of the tracer, and $i$, which gives the location of the tracer at any given time. We assume that when the tracer is at site $i$, it interacts with $\xi_{i}$. At a site $i$, $i \notin\{0, N+1\}$, the action is as follows: There is a clock which rings with rate $f$, for example $f=\eta^{-1 / 2}$ or $\left(\eta+\xi_{i}\right)^{-1 / 2}$, representing the time it takes for the tracer to make its way around the $i$ th box. When the clock rings, the following mixing of energies takes place [18]: Choose a random variable $p$ with uniform distribution in $[0,1]$. Then,

$$
\eta^{\prime}=p\left(\xi_{i}+\eta\right), \quad \xi_{i}^{\prime}=(1-p)\left(\xi_{i}+\eta\right)
$$

If $i=0$, then $\eta$ is replaced by a value chosen from the exponential distribution for the temperature $T_{\mathrm{L}}$. The rule at the right end $(i=N+1)$ is similar. After these operations, the tracer jumps with probability $\frac{1}{2}$ to $i-1$ or $i+1-$ except when it is at the ends, in which case it stands still with probability $\frac{1}{2}$ or moves into position 1 (resp. $N$ ) from the boundary. We prove in [8] that for all such models, the TP is linear and the distributions of the $\xi_{i}$ satisfy LTE.

Model II (Locked-in tracers): In this model there is a channel one-disk wide, with reflecting boundaries, and with turning disks located at fixed distances apart. These disks turn freely, but they block the channel completely, separating it into individual cells. Inside each cell is a single tracer, which moves back and forth, transferring energy between the turning disks that border the cell [19]. Here one can assume the tracer hits the two turning disks alternately, or, to further randomize the situation, one can add a number of fixed disks in each cell as illustrated in Fig. 2 After hitting one turning disk, the tracer then "gets lost" in this array of Lorentz scatterers, to emerge at some random moment to hit the turning disk at 


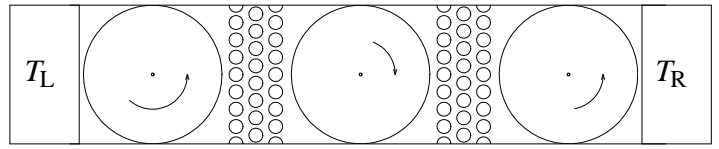

FIG. 2: A sketch of Model II, when it is made more chaotic. Between the rotating disks, there are disks serving as Lorentz scatterers. The tracers are not shown. The horizontal walls are reflecting.

either side with equal probability. In both cases, the time-offlight of the tracer between hitting turning disks depends only on the speed of the tracer (and not on the state of the disks).

Stochastic realization: We have $N$ sites on a 1-D lattice as in the previous stochastic model, with $\xi_{i}$ representing the energy of the turning disk at site $i$. In this model, however, there is one independent variable $\eta_{i}$ for each pair $(i, i+1), i=0, \ldots, N$, representing the energies of the tracers. Each site is equipped with an (independent) clock which rings at an exponential rate proportional to $\eta_{i}^{-1 / 2}$. When this clock rings, an exchange of energy involving $\eta_{i}$ takes place. As in the Hamiltonian model, one may assume $\eta_{i}$ exchanges energy alternately with $\xi_{i}$ and $\xi_{i+1}$, or, $\eta_{i}$ chooses with probability $\frac{1}{2}$ its left or right partner (i.e., $\xi_{i}$ or $\xi_{i+1}$ ), and performs the usual mixing: For example, if $\xi_{i}$ has been chosen, then

$$
\eta_{i}^{\prime}=p\left(\xi_{i}+\eta_{i}\right), \quad \xi_{i}^{\prime}=(1-p)\left(\xi_{i}+\eta_{i}\right)
$$

When the clock at site 0 rings, $\eta_{0}$ is replaced by a value chosen from the exponential distribution of temperature $T_{\mathrm{L}}$ as before. The rule at the right end $(i=N+1)$ is similar. Numerical simulations show clearly profiles deviating strongly from linearity. They are in perfect agreement with the value of $\alpha=\frac{3}{2}$ predicted by theory (see Fig 3 and the sketch of argument below).

The qualitative shape of the nonlinear profile can be understood easily by considering 3 successive sites, say $\xi_{i-1}, \xi_{i}$, and $\xi_{i+1}$. Since $\xi_{i-1}<\xi_{i+1}, \eta_{i+1}$ rattles faster than $\eta_{i}$ (the rate being given by $\eta^{-1 / 2}$ ). Thus $\xi_{i}$ equilibrates more often, and hence better, with $\xi_{i+1}$ than with $\xi_{i-1}$, which explains the concavity of the TP.

We next present some details of the theoretical arguments for the results above [8]:

Reduction from Hamiltonian to stochastic models: As dynamical systems, the Hamiltonian models above are very chaotic. With $\varepsilon$ in the MLL Model taken to be $\ll 1$, the dynamics are close to those in billiards of Sinai type and Lorentz gases. This chaotic behavior is used to induce a strong "memory loss" for the tracer particles. Fast correlation decay [16] ensures that the tracer "forgets" from which side it enters a site. This justifies our assumption in the stochastic model that the fraction of energy exchanged is independent from one step to the next.

Range of validity of (1). We consider a coupled chain of length $N$ in a steady state, and let $E_{i}$ be the mean temperature at site $i$. We assume (1) translation invariance of the model;

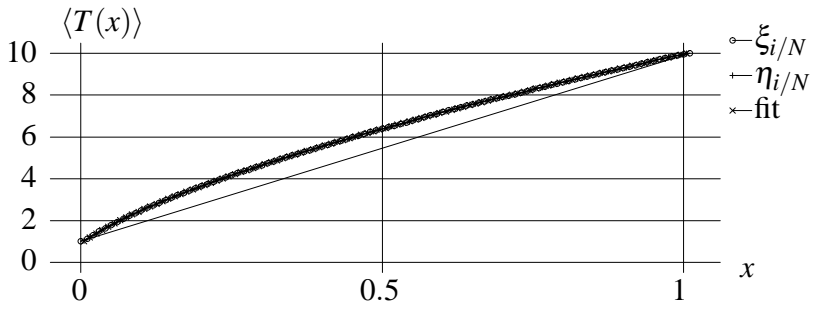

FIG. 3: The mean values of $\xi$ and $\eta$, as a function of $x=i / N$, with $T_{\mathrm{L}}=1, T_{\mathrm{R}}=10, N=100$, averaged over $2 \cdot 10^{9}$ exchanges of energy according to Model II. Superposed is the theoretical curve of Eq. 11. Note that the temperature profile is not linear and that its curvature is more pronounced at the cold end. Here, $\alpha=\frac{3}{2}$. We have obtained the same profile for many variants of Model II.

(11) $E_{i}$ is determined by $E_{i+1}$ and $E_{i-1}$; (111) energy-scale invariance, meaning if $E_{i \pm 1}$ are multiplied by $\lambda$, then $E_{i}$ is also multiplied by $\lambda$; (1v) as $N \rightarrow \infty$, the TP tends to a smooth function. It is not hard to show that (1)-(1v) imply that the limit stationary density satisfies a second order differential equation (namely 6), the solution of which with boundary conditions $T_{L}$ and $T_{R}$ is (1).

We demonstrate how to derive this law - and compute the constant $\alpha$ at the same time - for Model II:

Computing the temperature profile in Model II. Consider 3 successive sites. For illustration we assume (i) each tracer visits alternately the left and right disks and (ii) the mixing of energies at each collision is exactly half-and-half. The stationarity condition means that the speed of the tracer is equilibrated as well. Let the mean energy of the left tracer be $\eta_{-, \rightarrow}$ as it heads toward site $i$ and $\eta_{-, \leftarrow}$ as it goes away from it. By the rule of mixing, we have $\eta_{-, \rightarrow}=\left(\eta_{-, \leftarrow}+E_{i-1}\right)$, $\eta_{-, \leftarrow}=\left(\eta_{-, \rightarrow}+E_{i}\right)$, leading to $\eta_{-, \rightarrow}=\left(2 E_{i-1}+E_{i}\right) / 3$, $\eta_{-, \leftarrow}=\left(E_{i-1}+2 E_{i}\right) / 3$.

We assume the speed of the tracer is $E^{\gamma}$ when $E$ is its energy. The value of $\gamma$ for Model II as described above (and many other models without potential) is $\gamma=\frac{1}{2}$ (since the energy is purely kinetic) while for potential interactions, the time is given by an integral of the form $\int \mathrm{d} q(E-V(q))^{-1 / 2}$, which for large $E$ and $V(q) \sim|q|^{m}$ behaves like $\mathscr{O}\left(E^{-1 / 2+1 / m}\right)$, so that $\gamma=\frac{1}{2}-\frac{1}{m}$. Fixing $\gamma$, the average time for a round-trip of the tracer between sites $i-1$ and $i$ is $\tau_{-}=\eta_{-, \rightarrow}^{-\gamma}+\eta_{-, \leftarrow}^{-\gamma}$, and the rate at which site $i$ gets information from the left is the inverse of this quantity. An entirely analogous reasoning applies on the "+" side. From the stationarity condition, we get

$$
E_{i}=\frac{\tau_{-}^{-1}\left(E_{i}+\eta_{-, \rightarrow}\right) / 2+\tau_{+}^{-1}\left(E_{i}+\eta_{+, \leftarrow}\right) / 2}{\tau_{-}^{-1}+\tau_{+}^{-1}}
$$

Perform a perturbative analysis at the point $x=i / N$ where $N$ is very large. Then, to second order in $\varepsilon=1 / N, E_{i \pm 1}=$ $T(x) \pm \varepsilon T^{\prime}(x)+\frac{1}{2} \varepsilon^{2} T^{\prime \prime}(x)$, and (5) leads to

$$
t=T(x)+\varepsilon^{2} \frac{T^{\prime \prime}(x) T(x)+\gamma\left(T^{\prime}(x)\right)^{2}}{4 T(x)}+\mathscr{O}\left(\varepsilon^{4}\right) .
$$


Since $E_{i}$ must be equal to $T(x)$, we find

$$
T^{\prime \prime}(x) T(x)=-\gamma\left(T^{\prime}(x)\right)^{2},
$$

the solution of which with boundary conditions $T(0)=T_{\mathrm{L}}$ and $T(1)=T_{\mathrm{R}}$ is Eq. (1) with $\alpha=1+\gamma$. Thus for Model II, $\gamma=\frac{1}{2}$ and $\alpha=\frac{3}{2}$. One also checks that the energy flux is given by $T^{\prime}(x) \sqrt{T}(x)$ (which is constant along the profile, but not proportional to the temperature difference).

Generalization. Note that when $\gamma=0$, i.e., when the rate at which information is exchanged is independent of energy, then $\alpha=1$, which indeed gives a linear TP. Note also that our derivation is quite general: if $E^{\gamma}$ is replaced by $1 / F(E)$, the profile is given by $T^{\prime \prime}(x) F(T(x))=\left(T^{\prime}(x)\right)^{2} F^{\prime}(T(x))$.

Remark. Many authors have done careful simulations of models that are close to Model II, and have observed linear TPs. It should be noted that the profiles predicted by (1) are very close to linear when $T_{\mathrm{R}} / T_{\mathrm{L}}$ is not far from 1 . Our theory predicts that deviations from linearity become more prominent with the increase of $T_{\mathrm{R}} / T_{\mathrm{L}}$.

Linear profile and LTE for Model I. The reasoning above is not valid for Model I, for here it is a single tracer that is responsible for all transmission of information to all sites.

In the discussion below, we refer to the stochastic realization of Model I as a variable-time model, in the sense that the tracer lingers in each site for variable time periods depending on the function $f$. We introduce an associated fixed-time model, obtained by neglecting the waiting time above, i.e., all rules are as in the variable-time model except that the tracer jumps at fixed time intervals.

Linear profile. Consider first the fixed-time model. Here the tracer performs a standard random walk on $\{1,2, \ldots, N\}$ (except at the ends). It is easy to see that it spends an equal amount of time at each of the $N$ sites. Indeed for a fixed site $i$, the tracer comes from the left and the right exactly the same number of times over any period, for if the tracer heads left from site $i$, then it can only return from the left; and similarly for the right. Reasoning as above, we see that in the stationary measure, $E_{i}$ is affected equally by $E_{i-1}$ and $E_{i+1}$, hence the TP is linear, i.e., $\alpha=1$. Similar reasoning applies to the variabletime model. Note that the mixing rule, and hence the outcome of the mixing process, does not depend on the waiting time.

LTE. Methods similar to those in [10, 11] with a different dual XXX give LTE for the fixed-time model. While there is a correspondence between the sample paths for the variabletime model and its fixed-time counterpart, the varying durations the tracer spends at each site affects nontrivially the stationary measure. Proving canonical distributions for the model of interest, i.e., the variable-time model, requires further control of correlations [8]. Our proof applies to any function $f$ that is integrable and local, meaning it depends only on the variables $\xi_{i}$ and $\eta$ when the tracer is at site $i$ (which is the case here).

Remark: Even though this LTE result is for the stochastic realization of Model I, we have incorporated into our setting the notions of trajectories and velocities of particles, two of the key ingredients in Hamiltonian dynamics.

Conclusion. (1) We have pinpointed some simple and very natural mechanisms responsible for both linear and nonlinear profiles in homogeneous conductors modelled by Hamiltonian systems. Our results show in particular that when their ranges of motion are restricted, interacting particles (or springs) acquire energy-dependent speeds. (2) We have derived an exact formula - a universal law - for the energy profiles of very general Hamiltonian and stochastic chains with nearest-neighbor interactions. When nonlinear, this law predicts how deviation from linearity increases with the quotient $T_{\mathrm{R}} / T_{\mathrm{L}}$. (3) Finally, the underlying causes for nonlinearity that we have identified clearly go beyond the models studied here. They suggest that the presence of some (weak) nonlinear effect may be a more common phenomenon than recognized when very disparate temperatures are imposed at the two ends of a 1-D system.

Acknowledgments. We have profited from illuminating discussions with C. Mejía-Monasterio, D. Ruelle, L. ReyBellet, O. Lanford, Y. Avron and many others. JPE wishes to thank the Courant Institute and IHES for their kind hospitality. This research was partially supported by the Fonds National Suisse and NSF Grant \#0100538.

[1] J. B. J. Fourier, Oeuvres de Fourier / pub. par les soins de m. Gaston Darboux, sous les auspices du Ministère de l'Instruction Publique (Gauthier-Villars, Paris, 1888-1890).

[2] S. Lepri, R. Livi, and A. Politi, Phys. Rep. 377, 1 (2003).

[3] F. Bonetto, J. L. Lebowitz, and L. Rey-Bellet, in Mathematical physics 2000 (Imp. Coll. Press, London, 2000), pp. 128-150.

[4] B. Li, G. Casati, J. Wang, and T. Prosen (cond-mat/0307692).

[5] J. Hirschfelder, C. Curtiss, and R. Bird, Molecular Theory of Gases and Liquids (John Wiley, 1954).

[6] C. Mejía-Monasterio, H. Larralde, and F. Leyvraz, Phys. Rev. Lett. 86, 5417 (2001).

[7] H. Larralde, F. Leyvraz, and C. Mejía-Monasterio, J. Stat. Phys. 113, 197 (2003).

[8] J.-P. Eckmann and L.-S. Young, In preparation (2004).

[9] A. Dhar and D. Dhar, Phys. Rev. Lett. 82, 480 (1999).

[10] A. Galves, C. Kipnis, C. Marchioro, and E. Presutti, Comm. Math. Phys. 81, 127 (1981).

[11] C. Kipnis, C. Marchioro, and E. Presutti, J. Statist. Phys. 27, 65 (1982).

[12] H. Lorentz, Akademie van Wetenshaften te Amsterdam, Section of Sciences 7, 438 (1905).

[13] G. Casati, J. Ford, F. Vivaldi, and W. Visscher, Phys. Rev. Lett. 52, 1861 (1984).

[14] T. Prosen and M. Robnik, J. Physics. A 25, 3449 (1992).

[15] P. Garrido, P. Hurtado, and B. Nadrowski, Phys. Rev. Lett. 86 (2001).

[16] L.-S. Young, Ann. of Math. 147, 585 (1998).

[17] More than one tracer is sometimes used in [7], but we will limit ourselves to the single tracer case.

[18] Similar stochastic rules for mixing energies are used in [10, 11].

[19] A version of this model was suggested to us by D. Ruelle. 